\title{
Using Inquiry and Tree-Thinking to "March Through the Animal Phyla": Teaching Introductory Comparative Biology in an Evolutionary Context
}

\author{
James J. Smith • Kendra Spence Cheruvelil
}

Published online: 30 July 2009

(C) Springer Science + Business Media, LLC 2009

\begin{abstract}
Biodiversity was originally taught in our Introductory Organismal Biology course at Michigan State University (LB144; freshman/sophomore majors) by rote memorization of isolated facts about organisms. When we moved to an inquiry-based laboratory framework to improve pedagogy, an unfortunate and unforeseen result was the loss of much of our study of biodiversity. In this paper, we describe the restructuring of LB144 to restore the study of biodiversity and organismal groups while retaining the benefits of an inquiry-based approach. The curricular intervention was accomplished through the creation and implementation of a four-week Comparative Biology laboratory stream. During this stream, student research teams recorded and organized observations that they made on a range of organisms and analyzed their data in a phylogenetic framework. During the stream, our students worked through a set of exercises designed to help them learn how to read, interpret, and manipulate phylogenetic trees. We placed particular emphasis on the concept that phylogenetic trees are hypotheses of relationship that can be tested with scientific data. This incorporation of phylogenies and
\end{abstract}

J. J. Smith $(\bowtie) \cdot$ K. S. Cheruvelil

Lyman Briggs College, Michigan State University,

East Holmes Hall,

East Lansing, MI 48825, USA

e-mail: jimsmith@msu.edu

\section{J. J. Smith}

Department of Entomology, Michigan State University, 201 CIPS,

East Lansing, MI 48824, USA

\section{K. S. Cheruvelil}

Department of Fisheries and Wildlife, Michigan State University, 13 Natural Resources Building,

East Lansing, MI 48824, USA phylogenetic analysis, or "tree-thinking," into our students' work provided an explicit synthetic evolutionary framework for their comparative biodiversity studies. End-of-stream products included a team phylogenetic analysis exercise and an individual comparative biology oral presentation.

Keywords Comparative biology Evolution . Inquiry-based approach - Introductory biology . Phylogenetic analysis $\cdot$ Tree-thinking

\section{Background}

Biodiversity had been taught in our Introductory Organismal Biology course (LB144) for majors at Michigan State University (MSU) by rote memorization of isolated facts about organisms in the virtual absence of a synthetic evolutionary framework. Students came into the laboratory, observed a set of specimens (mainly preserved in jars) that represented the taxa of the week, drew pictures of them, and then memorized as many facts as possible about each specimen and its taxonomic group. This happened week-in and week-out for ten weeks, covering the range of eukaryotic taxa. One week, students would learn that whisk ferns are homosporous, while the next week they would learn that snails undergo torsion, and the next week that echinoderms have holoblastic cleavage. All of these activities culminated in a laboratory practical exam. Students indicated via written comments on course evaluations that satisfaction with this lab sequence was very low. Scores on the lab practical were also low, and our sense was that the students who succeeded on this exam did so by brute force memorization as opposed to deep learning.

One of the first changes we made to improve the LB144 lab experience was to move to an inquiry-based instruc- 
tional model (see Colburn 2000). During fall semester 2002, we adopted the "Teams and Streams" model of laboratory instruction (Wilterding and Luckie 2002). Using this model, students work in the lab in "teams" of three to four students. These research teams worked on inquirybased projects ("streams") of four to six weeks duration. In LB144, we developed one stream that focused on ecology, a second on genetics, and a third on developmental biology.

Unfortunately, this curricular change resulted in the loss of much of the basic study of organismal biodiversity that had previously formed a major focus of the course. This phenomenon is apparently not unique to our course, as McGlynn (2008) points out that this loss of biodiversity curricula is occurring in many places. So we asked, "how can we teach biodiversity in an inquiry-based framework?" We also wondered how we could do this without reintroducing the "passion-killing" activities, such as "draw what you see," during which students spent most of their time making superficial drawings of organisms they looked at either in jars of preservative or on prepared slides under a microscope.

The answer we came up with was to use phylogenetic analysis as the framework for one of our lab streams. There has been a strong movement recently to incorporate the use of phylogenetic trees to interpret evolutionary relationships, or "tree-thinking," into undergraduate biology education (Baum et al. 2005). A tree-thinking perspective can be taught as an element of evolution training at all levels and makes sense as an underlying framework for all biology. After all, a primary objective for this course is for our students to understand why it is that, "Nothing in biology makes sense except in the light of evolution" (Dobzhansky 1973).

We hypothesized that tree-thinking in the context of comparative biology would help our students understand and gain a better appreciation of natural history and the evolution of biodiversity. Several prominent scientists (e.g., Futuyma 1998; Grant 2000; Wilson 2000) have made pleas for stronger organismal training of college biology students (see McGlynn 2008). Therefore, we worked to provide our students with the foundation with which to understand organisms and how the environments in which they live have shaped them over evolutionary time.

This new LB144 lab stream, which we named the "Comparative Biology" stream, was derived from what had been the Developmental Biology stream. Specifically, we created a lab stream such that students would study and compare organisms in the context of other, related organisms with respect to their anatomy, morphology, physiology, and development. The Comparative Biology stream was designed to help students understand that (1) organismal diversity represents the result of an evolutionary process, (2) many shared characters among organisms (e.g., developmental, morphological) are present because groups of organisms are related to each other (i.e., they share a common ancestor), and (3) phylogenies represent hypotheses of evolutionary relationships. We addressed these objectives by instituting curricular changes to teach our students about organisms, not in a vacuum, but in a rich phylogenetic and evolutionary context.

In this paper, we describe the development and implementation of the Comparative Biology laboratory stream that we created for our Introductory Organismal Biology course. We describe the instructional elements that comprise the stream, our experiences with the implementation of the lab stream as part of our course, and a preliminary examination of its effects on student understanding and appreciation of organismal biology. We end with additional ideas for how the stream could be modified in the future and a description of our ongoing efforts to quantify the impact of these instructional activities on student learning.

\section{Our Course and the Context of the Curricular Change}

Lyman Briggs Introductory Organismal Biology (LB144) is a four-credit course with a total enrollment of 90-140 students per semester. Approximately $10 \%$ of the LB144 students are non-majority students. The majority of the students in LB144 are from suburban and rural Michigan. About half of our students take LB144 as freshmen, and half as sophomores. Our students come to us with a range of biology backgrounds. Many students have not had a biology course since ninth grade, while others have had two to three years of high school biology, and some have even taken Advanced Placement Biology.

LB144 is taught on a rotational basis by four LBC biology professors who share basic pedagogical views and a commitment to high teaching and learning standards. LB144 students attend two 80-minute lectures per week and a single three-hour session that serves as a combined recitation and lab. The lecture meets as a single section in which we employ a "bookends" instructional model (Johnson et al. 1998), with mini-lectures of ten to 15 minutes interspersed with small group exercises, individual writing, personal response pad questions, or other active/collaborative learning activities.

The combined recitation/lab meets in five or six sections with 18-24 students in each section. Lab sessions are staffed by a LB professor or a graduate Teaching Assistant (TA), plus two undergraduate learning assistants. Students in the labs work in teams of three to four students, yielding six to eight research teams per lab section. This model leads to a very favorable student/instructor ratio, especially when the research team is considered to be the student unit. 
In the LB144 lab, student teams work on three four- to six-week investigations, or streams. During a typical semester, an LB144 student team will complete an ecology, genetics, and comparative biology stream, each with their own end product. At the beginning of the first stream of the semester (often ecology during the fall and genetics during the spring), students learn about scientific reasoning, hypothesis formulation, and experimental design and statistical analyses. For the ecology stream, students devise and carry out an independent research project. Students present the results of their work at a poster session that mimics a professional scientific meeting. The genetics stream focuses on the study of sex-linked genes in Drosophila and Mendelian ratios in Wisconsin Fast Plants. Student teams write up their results in the format expected for a manuscript being considered for publication in a scientific journal.

Before the advent of the comparative biology stream (below), the third stream was a developmental biology stream, during which the students examined developmental sequences of sea urchins and the Japanese medaka and carried out a research project on regeneration in either planaria or blackworms. The end result of this stream was the construction of a web site. The regeneration research projects were problematic on several levels, as was web site construction. These, as well as some of the elements mentioned above (e.g., lack of study of organisms per se), were the elements of the lab that we sought to change by developing the comparative biology stream.

\section{The Comparative Biology Laboratory Stream}

\section{Overview of the Stream}

The new comparative biology lab stream was designed to last four weeks (however, see "Future Prospects and Conclusions" for modifications that can alter this timeline). Week one engages the students in an exploration of different animals, how we might study their similarities and differences in an organized way, and how these patterns are a result of evolutionary relationships as depicted by phylogenetic trees. These activities allow us to explore the idea of phylogenies as hypotheses of evolutionary relationship. During weeks two and three, student teams make a series of observations, both at the gross scale and the micro scale (using dissecting and compound microscopes, respectively), of live and preserved animals representing nine animal phyla that form the basis of their study. These observations include the dissection of four representative organisms. Teams record their observations in tabular form and supplement their observations with information available from literature sources. During week four, we conclude the stream with a team exercise that requires students to use their tabulated observations to demonstrate an understanding of phylogenies as hypotheses of relationship. In addition, each student gives a short oral presentation describing one aspect of his/her own animal explorations.

\section{Learning Goals}

We had two overarching goals for the comparative biology stream:

1. Introduce students to the natural history of animal groups by describing their basic features and naming a representative of each of the following nine animal phyla (and to be aware that there are other phyla): Porifera, Cnidaria, Platyhelminthes, Nematoda, Annelida, Mollusca, Arthropoda, Echinodermata, and Chordata.

2. Help budding biologists to understand evolution by applying a phylogenetic (tree-thinking) approach that uses phylogenetic trees as testable hypotheses of evolutionary relationships within and between groups of organisms.

In addition, we wanted to provide students with opportunities to use technology (e.g., digital photomicroscopy, MS PowerPoint) and practice effective oral communication skills.

\section{Descriptions of the Lessons}

Below we describe, in general, the lessons and activities that comprise the Comparative Biology stream. Detailed lesson plans and links to selected examples of student work are available in Appendices 1 and 2, respectively.

An Introductory Phylogeny Exercise in Lecture One of our major instructional goals for LB144 is to mesh the lectures and labs so that they work together to create a single learning experience. Therefore, we prepared our students for the comparative biology laboratory stream during lecture with a lesson that included a brief introduction to phylogenies and tree-thinking. This lesson was followed by an active learning exercise that allowed students to practice working with these concepts and allowed us (the teaching team) to assess their level of understanding.

One of the key ideas we try to get our students to understand in LB144 is that biology is an experimental science, and not just a collection of facts. Thus, we began the lecture with the concepts of a phylogeny as a hypothesis of evolutionary relationships and of a phylogenetic tree as a useful visualization tool to represent these hypothesized relationships (Fig. 1). We emphasized to the students that we can build phylogenies and trees using morphological, 


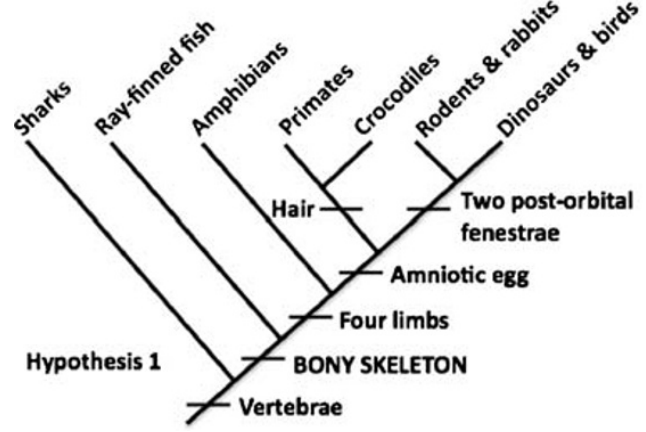

Fig. 1 Alternative hypotheses for the relationships of a set of vertebrate taxa, used to introduce students to the principle of parsimony as applied to phylogenetic analysis. In the two trees shown, we illustrate the concept by showing students that the bony skeleton is hypothesized to have arisen once in tree 1 , while tree 2 requires two separate evolutionary origins of this character state. Students are subsequently

developmental/embryological, and/or life history features, and that it is now common to use molecular biology (e.g., DNA sequences) to make inferences about phylogenetic relationships. We also emphasized that every phylogeny represents a hypothesized answer to the questions, "Which of the taxa being studied share relatively recent common ancestors? Which share relatively distant ancestors?"

As a hypothesis, every phylogeny can then be evaluated on the basis of evidence (i.e. what is the evidence in favor of or against a particular phylogenetic tree?). After practicing how to read phylogenetic trees, including a presentation of material on how relative time is incorporated into a phylogenetic analysis, we discussed ways to evaluate alternative hypotheses (trees). This discussion included mapping characters onto trees (where a particular character state would have evolved or been lost on a tree) and using the Principle of Parsimony (i.e. the simplest tree, with the fewest number of evolutionary events, is more likely to be the correct tree) to choose between two competing hypotheses (Fig. 1). By the end of this lesson, the students were primed to actively explore these ideas and assess their understanding of phylogenies.

We ended this portion of the class with an active learning exercise that had students work both individually and with a partner to evaluate alternative hypotheses of evolutionary relatedness using hypothetical molecular data. This exercise uses three trees (Fig. 2, trees I, II, \& III) that depict relationships between four hypothetical species (Fig. 2 AD). Our goals for this exercise were for the students to understand and practice evaluating alternative hypotheses (trees) and for us to assess their level of understanding.

This lecture and in-class exercise nicely set the stage for the comparative biology lab stream that was to begin later that week. The students were introduced to phylogenies as hypotheses of evolutionary relationships and the ways that phylogenetic trees are constructed and evaluated using

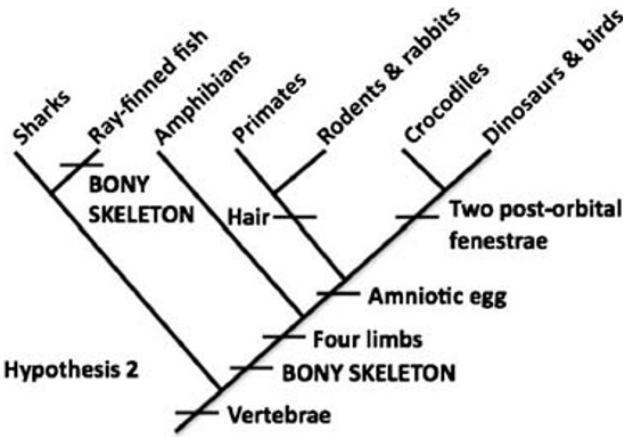

given an exercise in which they are challenged to count the number of evolutionary changes in each of two trees, and then decide which of the two trees is a better phylogenetic hypothesis. Graphic illustration adapted with permission from the University of California's Understanding Evolution Web Site (http://evolution.berkeley.edu/evolibrary/ article/0_0_0/phylogenetics_08)

scientific data. This knowledge, along with the experience the students gained during the active learning exercise, led directly into the first week's comparative biology lab activities that had students collect and organize scientific data in order to think about similarities and differences among organisms and use scientific data to evaluate alternative hypotheses (trees). In addition, the active learning exercise's use of molecular data to evaluate alternative trees was a nice complement to the lab activities that use morphological, developmental, and life history data to evaluate alternate hypotheses of evolutionary relationship.

Introductory Exercises in the Lab We began week one of the comparative biology stream with an engagement

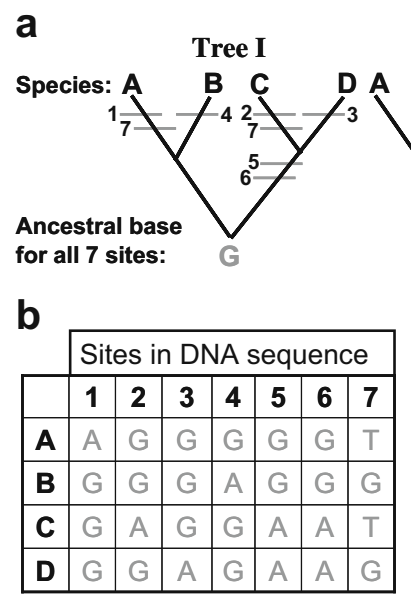

Fig. 2 Graphic illustrations used in an active learning exercise in lecture during which students evaluate alternative evolutionary hypotheses (adapted from Campbell and Reece 2002). a Trees I-III show different evolutionary relationships between four hypothetical species $(A-D)$; b states for seven molecular characters for the four species. Tree I is marked with eight labeled horizontal lines that represent evolutionary changes in these seven characters (i.e., DNA base changes) 
exercise. An array of living and preserved organisms (Table 1) was scattered throughout the lab at different workstations (see Online Supplemental Materials for the complete week one lesson plan in Appendix 2). Our goal was to have the students discover how to build and use a taxon/characteristic state matrix. Microscopes were provided and students were instructed to spend a total of 20 minutes observing the 18 organisms that were on display. In addition, students were instructed to take notes, listing any observations and/or questions that their team had. Once the observation session was complete, the student teams put their observations into a logical framework in the form of a data table. We helped them by asking, "What will you use as rows? Columns? (Why?)" We then debriefed the exercise with a short in-class discussion during which we highlighted contrasting ways to arrange the data collected using the organisms.

Next, we took advantage of and adapted two published studies that used inquiry and phylogeny in the biology classroom (Singer et al. 2001; Giese 2005). Singer et al. (2001) introduced the concepts of a characteristic table, cladistic analysis, and character conflict and demonstrated how to use these concepts to compare competing phylogenetic hypotheses. The authors were inspired in part by the work of Bilardello and Valdés (1998), whose article on building phylogenies showed how doing a phylogenetic analysis provides practice in critical thinking, strengthens students' logical and mathematical abilities, and strengthens their problem-posing and problem-solving skills. Giese (2005) described a classroom exercise during which student teams were given "incorrect" phylogenies for a number of taxa for which they had available fully articulated skeletons. Students were then challenged to find skeletal evidence to reject each hypothesis, using "If-Then" reasoning. We fused aspects of these exercises from Singer et al. (2001) and Giese (2005) to create our own exercise that allowed students to practice making and testing alternative phylogenies.

For this exercise, we provided student teams with fully articulated skeletons and bone keys from each of the five taxa that were the focus of Singer et al. (2001; Fig. 3). Student teams were challenged to study the five skeletons and make informal comparisons by noting similarities and differences between them. Student teams were then provided with a table containing characters of each of the five taxa (Table 2), asked to find three to four characters (one per person) that could be used to group taxa together to the exclusion of other taxa and challenged to see if they could

Table 1 Laboratory organisms studied in the Comparative Biology stream

\begin{tabular}{|c|c|c|c|c|c|}
\hline Scientific name & Common name & Phylum & Live/preserved & Engagement & $\begin{array}{l}\text { Phylogenetic } \\
\text { comparison }\end{array}$ \\
\hline Scypha sp. & Sponge & Porifera & Preserved specimen; prepared slides & $\mathrm{X}$ & $\mathrm{X}$ \\
\hline Gonionemus sp. & Gonionemus & Cnidaria & Preserved specimen & $\mathrm{X}$ & \\
\hline Hydra sp. & Hydra & Cnidaria & Live specimen & $\mathrm{X}$ & $\mathrm{X}$ \\
\hline Hexagonaria percarinata & Petoskey stone & Cnidara & Fossil & $\mathrm{X}$ & \\
\hline Dugesia tigrina & Planarian & Platyhelminthes & Live specimen; prepared slides & $\mathrm{X}$ & $\mathrm{X}$ \\
\hline Dipylidium caninum & Dog tapeworm & Platyhelminthes & Preserved specimen & $\mathrm{X}$ & \\
\hline Ascaris lumbricoides & Roundworm & Nematoda & $\begin{array}{l}\text { Preserved specimen for dissection; prepared } \\
\text { slides }\end{array}$ & $\mathrm{X}$ & $\mathrm{X}$ \\
\hline Nereis succinea & Clamworm & Annelida & Preserved specimen & $\mathrm{X}$ & \\
\hline Lumbricus terrestris & Earthworm & Annelida & Live specimen; preserved specimen for dissection & $\mathrm{X}$ & $\mathrm{X}$ \\
\hline Romalea sp. & Grasshopper & Arthropoda & Preserved specimen for dissection & $\mathrm{X}$ & $\mathrm{X}$ \\
\hline $\begin{array}{l}\text { Gromphadorhina } \\
\text { portentosa }\end{array}$ & $\begin{array}{l}\text { Hissing } \\
\text { cockroach }\end{array}$ & Arthropoda & Live specimen & $\mathrm{X}$ & \\
\hline Cambarus sp. & Crayfish & Arthropoda & Preserved specimen for dissection & & $\mathrm{X}$ \\
\hline Anodonta sp. & $\begin{array}{l}\text { Freshwater } \\
\text { mussel }\end{array}$ & Mollusca & Preserved specimen for dissection & $\mathrm{X}$ & $\mathrm{X}$ \\
\hline Loligo pealei & Squid & Mollusca & Preserved specimen & $\mathrm{X}$ & \\
\hline Pisaster sp. & Sea star & Echinodermata & Live specimen; Preserved specimen & $\mathrm{X}$ & $\mathrm{X}$ \\
\hline Eupentacta sp. (?) & Sea cucumber & Echinodermata & Live specimen & $\mathrm{X}$ & \\
\hline Strongylocentrotus sp. & Sea urchin & Echinodermata & Live specimen & $\mathrm{X}$ & \\
\hline Branchiostoma sp. & Lancelet & Chordata & Preserved specimen; whole mount & $\mathrm{X}$ & $\mathrm{X}$ \\
\hline Rattus rattus & Rat & Chordata & Preserved specimen & $\mathrm{X}$ & \\
\hline
\end{tabular}

Also indicated is whether the organism was used as a live or prepared specimen and whether or not it was used in the engagement exercise (week one) and/or the phylogenetic comparison (weeks two to four) 
Fig. 3 Photographs of the skeletons of the opossum (a), dog (b), rat (c), cat (d), and rabbit (e). These skeletons were used by the students to explore the basis of characters and help learn how to build and interpret a taxon/character state matrix

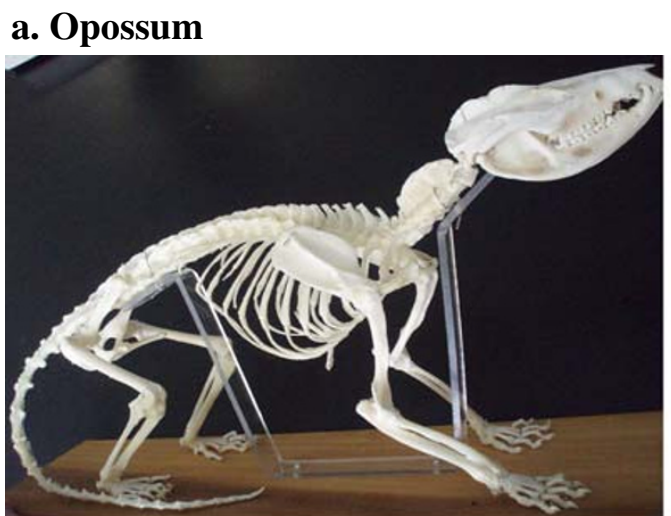

\section{d. Cat}

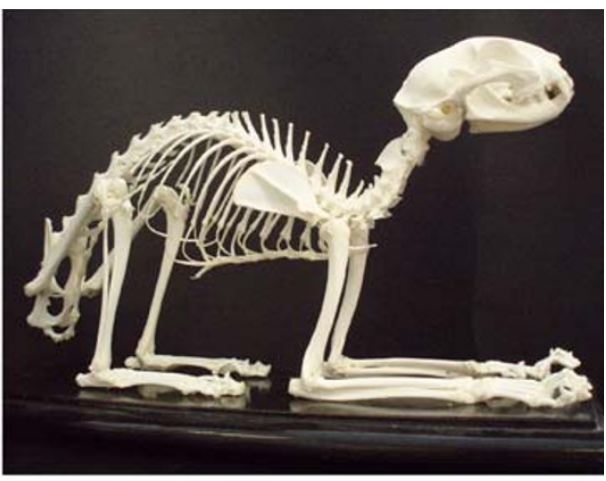

b. Dog

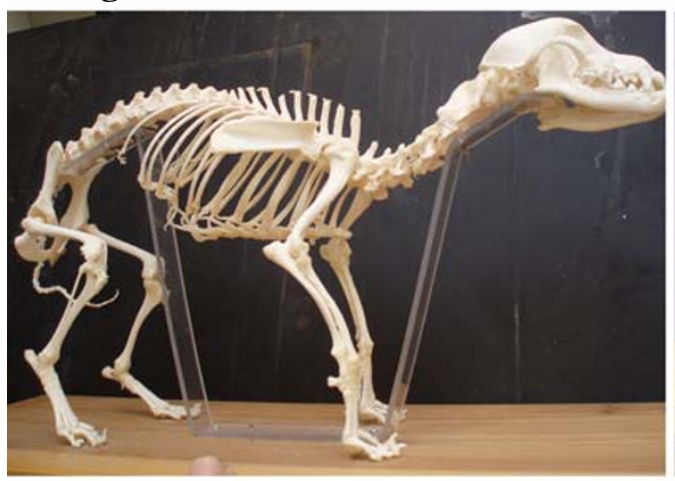

e. Rabbit

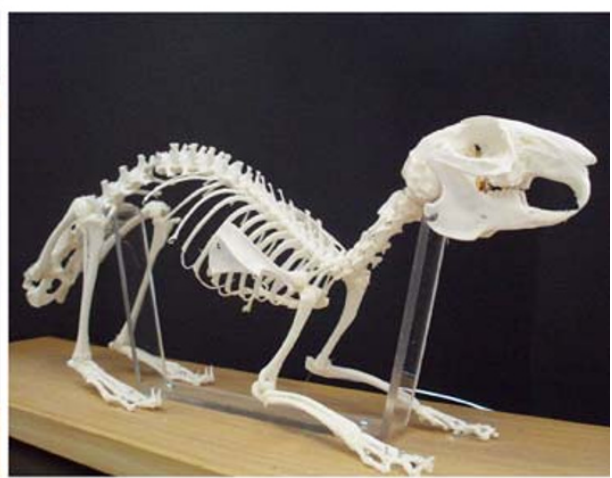

c. Rat

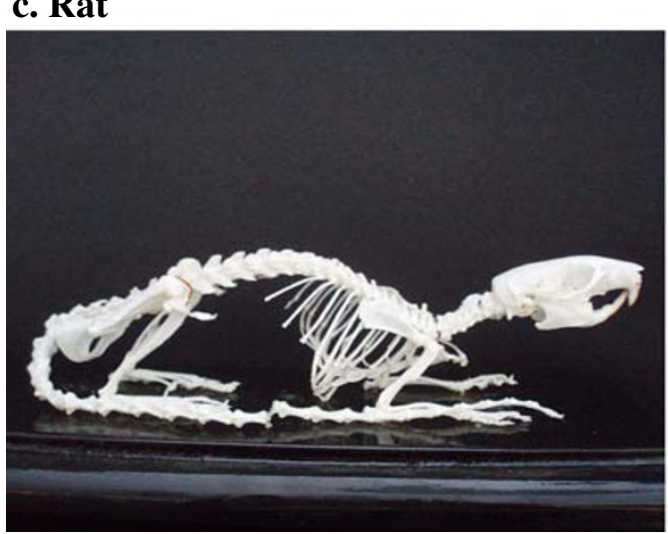

discover additional characters on their own by using information on each animal available through the University of Michigan Museum of Zoology's Animal Diversity Web (http://animaldiversity.ummz.umich.edu/site/index. html). Finally, students were asked to reflect on which characters in the dataset they thought might be most useful for evaluating phylogenetic hypotheses, taking into consideration the animals' physiology (e.g., Does it feed the fetus via a placenta?), behavior (e.g., Is it a social animal?), and ecology (e.g., Is it an aquatic animal?).

The capstone experience for week one was for the students to compare two arbitrarily chosen trees of the 15 possible rooted trees for these five taxa (Fig. 4). We first asked the students to convince themselves that only 15 rooted phylogenies are possible for five taxa (with opossum as the outgroup), and that one of these must be the true phylogeny. Students were guided in this lab activity towards developing an argument based on the characters in their table, and/or any characters that they had discovered by observing the skeletons. Student teams worked together to analyze the evolution of the set of characters in their table for each phylogenetic tree. Based on this analysis, they then explained to the class which of the two phylogenies they thought was a better hypothesis. This exercise nicely linked with the active learning exercise conducted earlier in the week during lecture (above; Fig. 1) and provided students more practice using scientific data to evaluate different phylogenetic trees as alternative hypotheses. 
Table 2 Table of characters observed in five mammals (adapted from Singer et al. 2001)

\begin{tabular}{|c|c|c|c|c|c|}
\hline Character & Opossum $^{\mathrm{a}}$ & $\operatorname{Dog}^{b}$ & $\mathrm{Rat}^{\mathrm{c}}$ & $\mathrm{Cat}^{\mathrm{d}}$ & Rabbit $^{\mathrm{e}}$ \\
\hline Placental & - & + & + & + & + \\
\hline Prehensile tail & + & - & - & - & - \\
\hline Solitary lifestyle & + & - & + & + & $+/-^{\mathrm{f}}$ \\
\hline No. of teeth & 50 & 42 & 16 & 28 & 28 \\
\hline Large canine teeth & + & + & - & + & - \\
\hline Large incisors & - & - & + & - & + \\
\hline Expanded metatarsals & - & + & - & + & + \\
\hline Hopping locomotion & - & - & - & - & + \\
\hline
\end{tabular}

Instructions to students: Some characters are provided and scored for you, others you need to identify and score yourselves using the five mammal skeletons and keys (one character per person). You may also use the web sites below to collect additional natural history data on the organisms to help you evaluate the two hypotheses (phylogenetic trees)

${ }^{a}$ http://animaldiversity.ummz.umich.edu/site/accounts/information/Didelphis_virginiana.html

${ }^{\mathrm{b}} \mathrm{http} / / /$ animaldiversity.ummz.umich.edu/site/accounts/information/Canis_lupus_familiaris.html

${ }^{\mathrm{c}} \mathrm{http}$ ://animaldiversity.ummz.umich.edu/site/accounts/information/Rattus_rattus.html

${ }^{\mathrm{d}} \mathrm{http} / / /$ animaldiversity.ummz.umich.edu/site/accounts/information/Felis_silvestris.html

${ }^{\mathrm{e}} \mathrm{http}$ ///animaldiversity.ummz.umich.edu/site/accounts/information/Oryctolagus_cuniculus.html

${ }^{\mathrm{f}}$ Variation within the group precludes categorizing rabbits as solitary or social

Observations and Dissections During weeks two and three of the comparative biology stream, students collected data from a set of ten organisms representing nine animal phyla. These lessons, which involved observations on all ten organisms and four key dissections (roundworm, earthworm, crayfish, and grasshopper), were adapted from Lab Topics $17 \& 18$ in Morgan and Carter (2005). We found the dissection guides presented in Morgan and Carter (2005) to be particularly useful. These two lab topics were included in their entirety in the LB144 lab book that each student was required to purchase for the course. In the laboratory itself, each of the student research teams also had available a brief guidebook to the ten animals that they were studying, consisting of selected pages from Elson (1982) and Rust (1983). In addition, we assigned a pair of our undergraduate learning assistants in the course to take the lead on each of the four dissections by preparing a brief guide for the students to follow and demonstrating the dissection to the whole teaching team prior to lab.

During these two lab weeks, student teams observed each of the ten animals both visually, to see gross anatomical features, and with dissecting microscopes to observe finer structural detail. For each animal observed, the teams took a set of photographs to document observations using microscopes equipped with digital cameras. For some animals, students were also provided with prepared microscope slides that were also used for observation via digital photomicroscopy (e.g., the planarian, the roundworm, and the earthworm). The use of digital photomicroscopy provided a simple way for students to record data from their organismal observations. By encouraging students to record data according to their own interpretations of what was important, we hoped to provide a sense of ownership of their animal investigations.

A casual observer may have come away from observing these two weeks thinking that our students simply did the labs in Morgan and Carter (2005). For example, during these two weeks of observations, the student teams filled out a summary table adapted from Table 18.1 in Morgan and Carter's text (Table 3). However, we also included treethinking activities in our students' investigations during these two weeks to get them thinking about their observations in an evolutionary context (see Concluding Exercises, below).

Another major goal of the comparative biology stream was to have student teams create an electronic notebook containing all of their observations of the ten animals. This notebook took the form of a PowerPoint file that contained all of the student pictures taken during weeks two and three. Each team was required to establish a collection of four to six slides per animal (some with multiple photos per slide). Each photo was annotated with labels, legends, and text explaining what the specimen was, how it was observed (whole or mounted on a microscope slide), what type of sectioning was used (whole mount, cross section, longitudinal section), and whether it was observed with a dissecting or compound microscope (and at what total magnification). A link to an example of one such electronic notebook is included in Appendix 2.

Concluding Phylogeny Exercises in Lab and Lecture There were two main activities during week four of the 
Fig. 4 Fifteen possible phylogenetic trees for rat, rabbit, dog, and cat, rooted with opossum as the outgroup. Student teams are given two arbitrarily chosen trees and asked to use data to decide which of the two trees is a better hypothesis

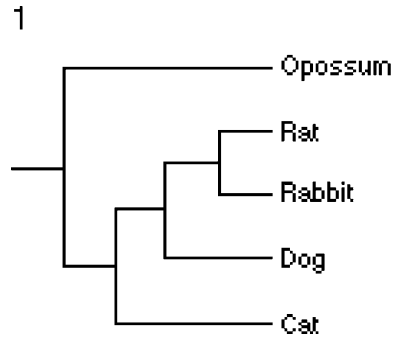

4

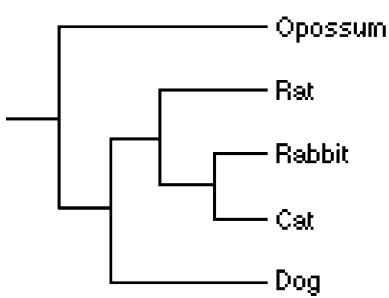

7

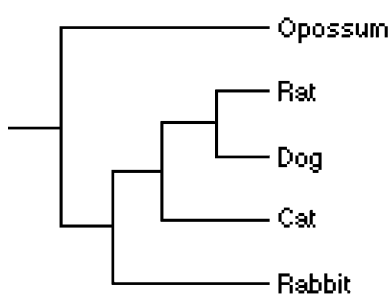

10

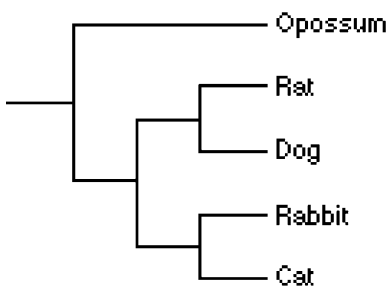

13

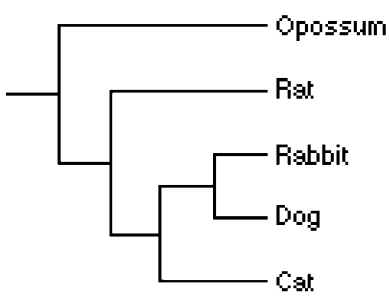

2

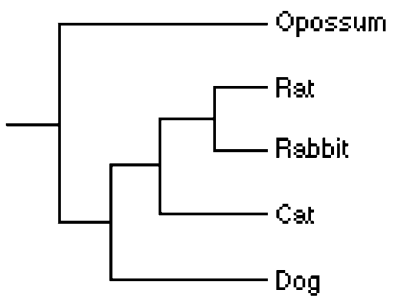

5

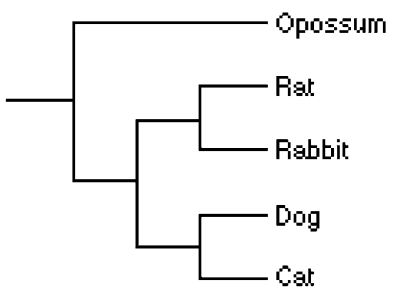

8

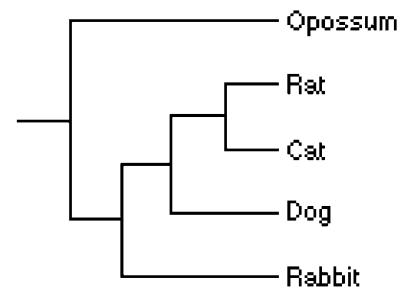

11

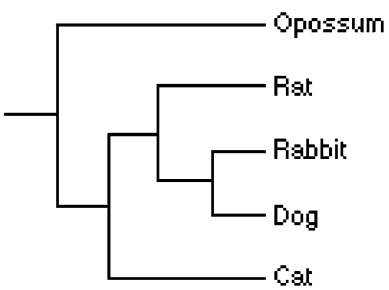

14

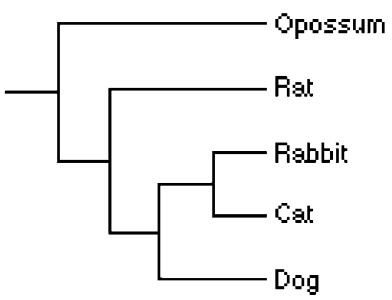

3

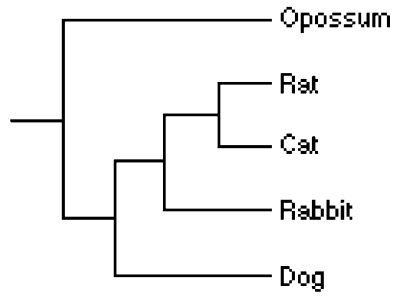

6

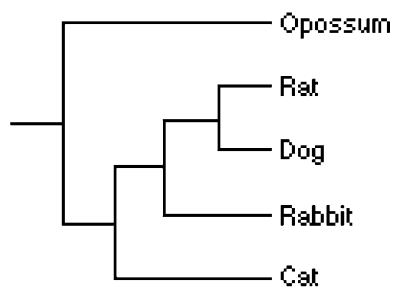

9

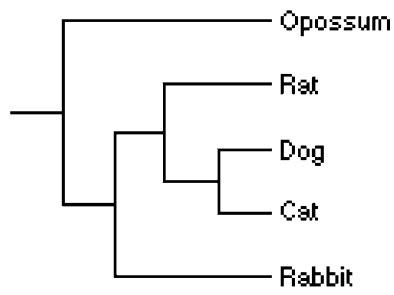

12

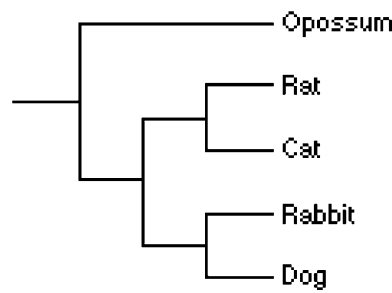

15

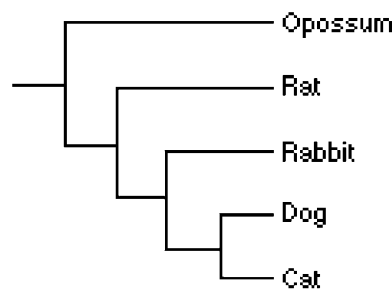

comparative biology lab stream. First, student teams were given a team assignment called, "Mapping Characteristic Evolution on Competing Phylogenies" to bring together the information collected during weeks two and three with the techniques that they learned and practiced evaluating evolutionary hypotheses (i.e., phylogenies) during week one of the stream and during lecture. The idea was to have the students use evidence that they themselves had collected to map characteristic evolution onto two phylog-

enies (Fig. 5) and to evaluate the relative strength of the two phylogenies, much as they had done in week one of the stream.

Second, each individual student was required to organize a set of two PowerPoint slides that would form the basis of an oral presentation that they would give to the class during week four. Each of these slides included multiple photos that demonstrated student understanding of how at least three of the animal phyla observed are similar and different. 


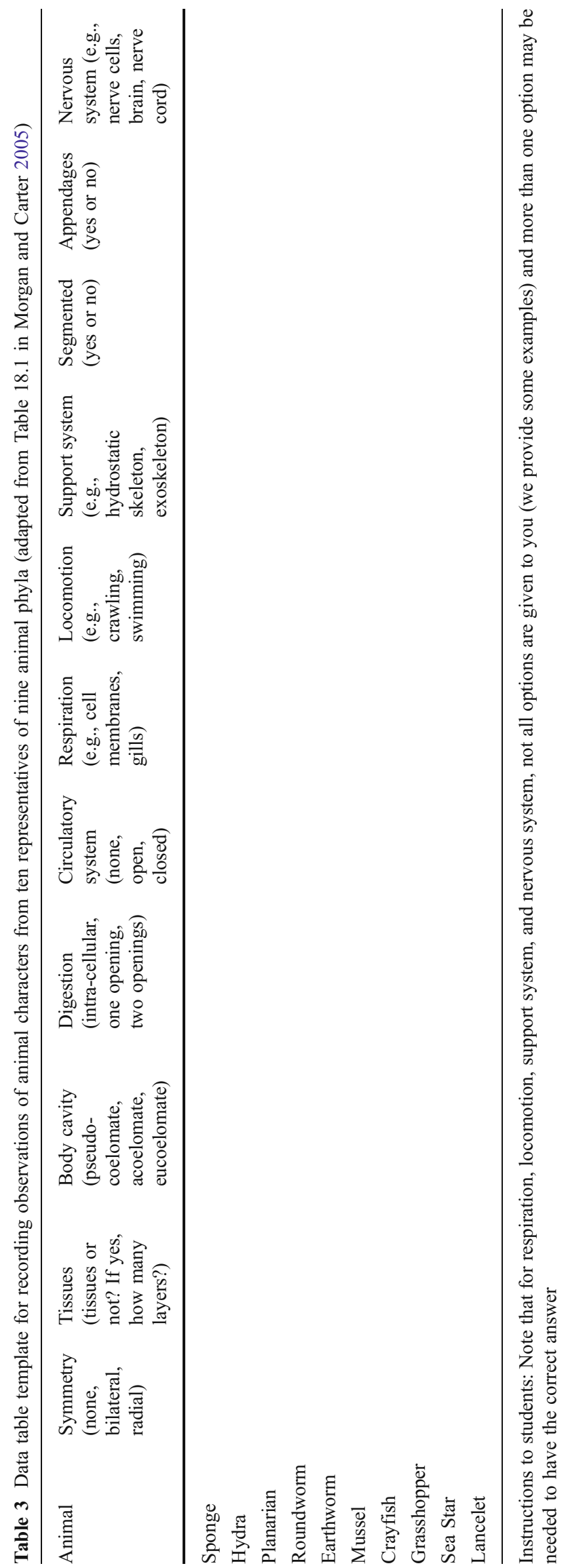

For example, a student might show how the respiratory system differs across three animal phyla and talk about what that means in a phylogenetic context. A link to a file containing examples of slides produced by LB144 students is included in Appendix 2. These presentations were to mimic those found at a scientific conference, allowing students to practice their oral communication skills. In addition, this exercise provided the students with the opportunity to demonstrate individual knowledge and understanding of the comparative biology concepts.

\section{Implementation of the Stream and Assessment of Student Learning}

We implemented the comparative biology stream during fall semester 2006 (F06) and spring semester 2007 (S07) using the instructional sequence described above. During F06, assessment of student learning in the comparative biology stream included a lab quiz that had a question about mapping characters and mirrored the work the students had done with the skeletons during week one of the stream. In addition, the LB144 final exam included a section on comparative biology. One of the extended response questions on this exam asked students to map the following characters onto a phylogenetic tree that had phylum names as terminal taxa: (a) true tissues; (b) radial and bilateral symmetry; (c) acoelomate, pseudocoelomate, and eucoelomate body plans; and (d) protostome and deuterostome embryological development. This exam question was almost identical to the question/task completed as a lab team during week four of the comparative biology stream (Fig. 5).

During S07, assessment of student learning included a lab quiz. However, we also gave a stand-alone hour exam that included a section on comparative biology. Our (the teaching team's) response to this exam was a sense that the students still did not "get it" with respect to mapping specified characters onto morphology-based trees. Therefore, in preparation for the final exam, we prepared an Animal Problem Study Guide that we handed out in class (not shown). We then asked the mapping question again on the final exam, but without the requirement to map the acoelomate, pseudocoelomate, and eucoelomate body plans (Fig. 5).

Student learning of tree-thinking apparently was better during S07 than S06. On the F06 final exam, the students scored an average of $3.86 / 6.00( \pm 1.66)$, or $64.3 \%$, on the characteristic-mapping question (Fig. 5); during S07, the mean score was $4.45 / 6.00( \pm 1.76)$, or $74.2 \%$. Among the possible reasons for the higher scores observed during S07 than F06 are the extra coaching and the second chance provided the students (hour exam and the final exam), and the easier question given on the final exam (not having to 


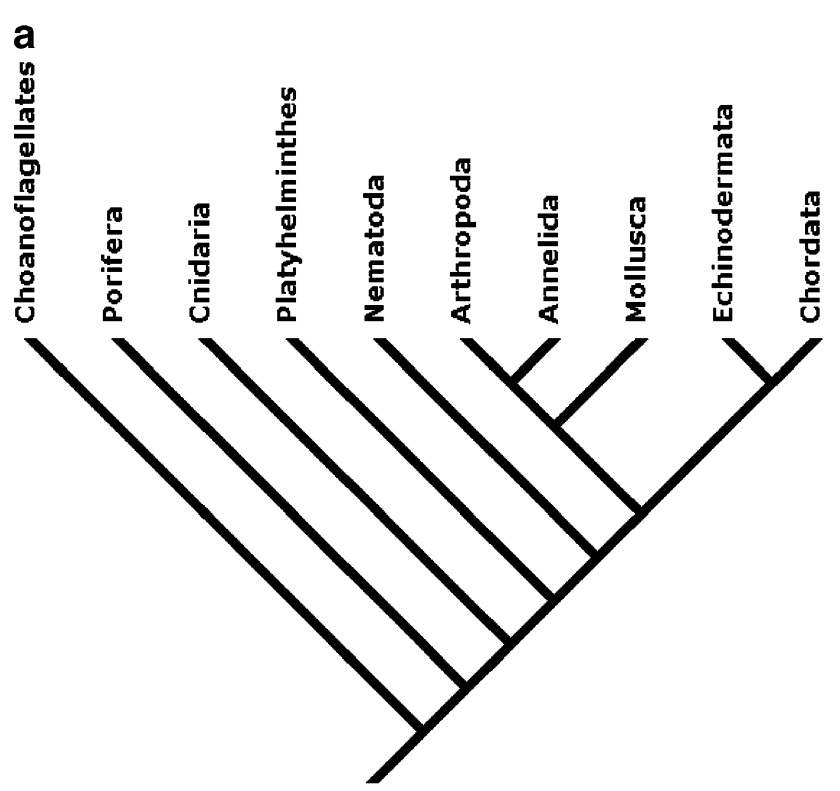

Fig. 5 Phylogenetic tree showing the hypothesized relationships for nine animal phyla. a Inferred from morphological and developmental characters (based on Freeman 2005, Figure 31.10); b inferred from molecular characters (based on (Freeman 2005), Figure 31.13). In a closed book laboratory exercise, students are asked to map each of three characters onto each of these two trees. The characters are: (1) body cavity (acoelomate, pseudocoelomate, or eucoelomate), (2) number of

map acoelomate, pseudocoelomate, eucoelomate; Fig. 5). We also do not know if there were differences in the student populations between the two semesters, whether additional instructional differences existed, or how well our assessment techniques accurately quantified student learning.

Our course evaluations at the end of the semester allowed us to obtain anecdotal student responses regarding the comparative biology lab stream. Several students commented that they really enjoyed the dissections. This comment makes sense given that many LB144 students are planning careers in medicine. Another sentiment expressed was that students appreciated the opportunity to demonstrate individual knowledge through the PowerPoint presentations.

Although we have presented here an informal preliminary analysis of student learning, formal quantitative assessment of student learning in the comparative biology stream is ongoing. We created and employed a Phylogeny Assessment Tool to assess prior knowledge (Pre-test) and learning outcomes (Post-test), and collected data from approximately 200 LB144 students during fall semester 2008. Data analyses are in progress and will form the basis of a separate manuscript (Smith and Cheruvelil, in preparation).

\section{Future Prospects and Conclusions}

We have presented here a four-week lab module supplemented by lecture activities and assessment, focusing

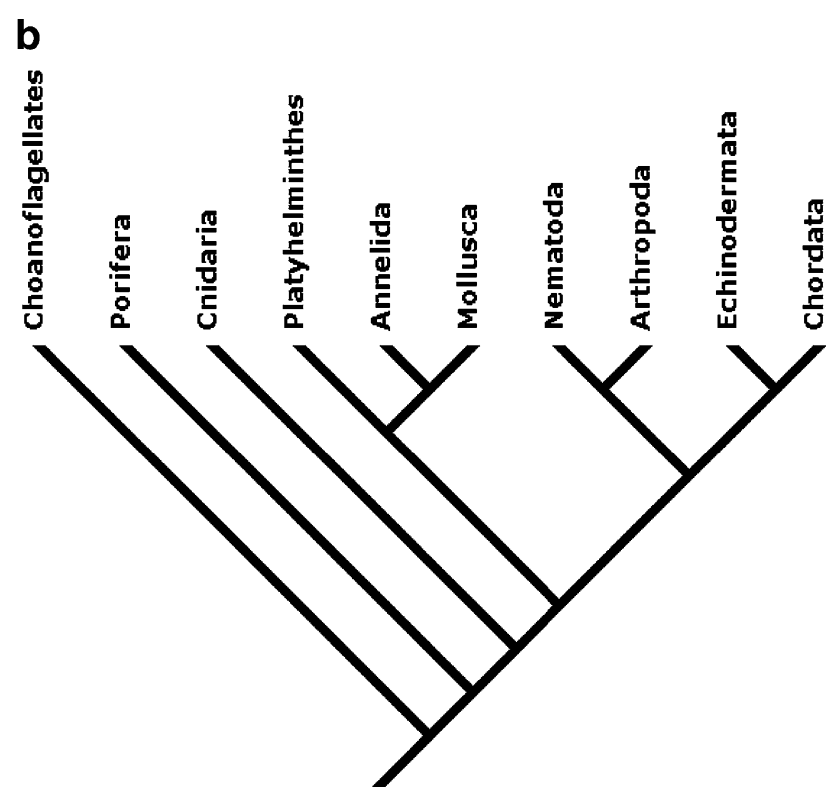

opening to the digestive tract (one or two), and (3) segmented body (yes or no). As part of a lecture exam, students are asked to map each of four characters onto tree (a). The characters are: (1) body cavity (acoelomate, pseudocoelomate, or eucoelomate; the question asked on the Final Exam during S07, for which we have data, omitted asking about body plans), (2) embryological development (protostome or deuterostome), (3) true tissues, and (4) symmetry (radial or bilateral)

mainly on the comparative morphology of animal groups. However, these materials can be adapted to fit other focus areas or groups. For example, we have incorporated an additional lab week into our own comparative biology stream, between weeks two and three, during which students compare embryological development in sea urchins, frogs, and the Japanese medaka. Results from some of these observations are included in the Online Supplemental Materials (see Appendix 2).

This laboratory stream could be adapted to plant systems, protists, fungi, and/or bacteria, and plants may be particularly well suited for this set of exercises. Plants have good morphological and developmental features that can be used in phylogenetic analysis; there is a good fossil record of plant evolution; and the phylogenetic relationships of the major plant groups are well supported by data. Alternatively, another simple way to incorporate plants into the lab stream would be to use an array of plant specimens instead of animals in the engagement exercise during week one of the stream.

There are some ways in which the lab stream could be modified and/or extended to deepen student understanding of evolution as an organizing principle. For example, once students become familiar with phylogenies and phylogenetic analysis, it should be possible to use phylogenies as a framework for advanced exercises in later parts of the organismal biology laboratory course, with students using phylogenies to test developmental, ecological, and/or evolutionary hypotheses. However, given some of the 
constraints of the introductory biology laboratory curriculum, these advanced exercises may be more appropriate for inclusion as laboratory components in upper division courses in ecology, developmental biology, and evolutionary biology.

From a tree-thinking perspective, Novick and Catley (2007) showed that students have an easier time correctly interpreting phylogenetic trees that are not "ladderized." The trees that we have used in the exercises discussed in Figs. 1, 2, and 5 are shown as ladders (slanted cladograms), and in future offerings of the LB144 comparative biology stream, we plan to modify these exercises by incorporating rectangular cladograms into them.

Another positive change would be to take more time during the lab stream for students to reflect on their learning through the use of pre-class quizzes and their student electronic notebooks. For example, we could use a pre-quiz such as one from The Tree-thinking Challenge (Baum et al. 2005) to help set the context during our introductory phylogeny lecture and provide students with a chance to reflect on their own understanding during and after the active learning exercise. We could also expand the use of the electronic notebooks by asking students to describe how their thinking has changed by virtue of carrying out this set of exercises.

Finally, it would be worthwhile to figure out how to incorporate molecular phylogenetic trees into the mix in a meaningful way. This is not a trivial problem. The standard tree that we used in our test questions is "parsimonious" with respect to the morphological features and developmental patterns of organisms typically taught in undergraduate biology. Unfortunately, a series of phylogenetic analyses of DNA sequences beginning in the late 1980s (Field et al. 1988) showed convincingly that this "most parsimonious' solution is not correct, and that morphological and developmental evolution in this group of organisms has occurred in a non-parsimonious fashion. Conveying this idea without causing student confusion is a major challenge. One idea might be to start the students on a smaller-scale exercise than the one we now do in the lab (Table 3; Fig. 5). Such an exercise could start with a series of organisms and a tree for which parsimonious morphological phylogeny appears to be accurate, but is not supported by molecular characters (nucleotide or amino acid characters). This exercise would illustrate the point that morphology does not always evolve in a parsimonious manner and provide a way to explore homoplasy and the nature of molecular evidence, without interference from the potentially overwhelming set of data collected from the nine animal phyla.

An additional problem arising from the construction of phylogenies from molecular data is that students are often encouraged to use similarity-based arguments to group organisms together. On the surface this is OK, and it does tend to give the "correct answer." However, from a "learning about the nature and practice of science" standpoint, this may not be such a good idea. In the comparative biology lab stream just described, we work very hard to help students understand that when two phylogenies are under consideration, they represent explicit, testable, alternative hypotheses of evolutionary relationship that can be evaluated with data (Avise 2004). This is the real strength of the character state approach, and other phylogenetic approaches do not lend themselves quite as easily to such a rich interpretive framework.

Phylogenies and phylogenetic analysis are powerful tools for helping students understand evolution. The use of phylogenetic trees to interpret evolutionary relationships, or tree-thinking, has the potential to provide a synthetic evolutionary framework for biodiversity studies in undergraduate biology courses. This evolutionary framework is essential if we really want biology to "make sense" to our students. Training in natural history is also very important for our students' biology education, and many are finding that this type of training is more and more difficult to provide. Phylogenies can help in this instance because they can help put the study of organisms into an inquiry-based framework. The comparative biology lab stream that we have presented here builds upon the work of others to bring together tree-thinking and comparative biology of animals to help students understand animal biodiversity in an evolutionary framework and in an inquiry-based fashion.

Acknowledgments We thank Dr. Sue Hill (MSU Zoology) for initial discussions and support for the curricular change. Also, thanks to Lyman Briggs undergraduate students Parita Shah, Phil Timko, Lauren Doherty, and Courtney Ford, who participated in an Honors option in LB144 during spring 2006 when we laid the groundwork for much of the curricular change. We thank Lyman Briggs undergraduate students Sara Steslicki, Jake Carpenter, Nick Vito, Ryan DasGupta, Theresa Seitz, Megan Swiss-Schuster, Eliisa Bojanic, Lisa Rousseau, Lauren Priniski, Krystal Krejcik, Victoria Roney, Cassie Martin, Greg Klein, Samantha Steffen, Ashley Lee, Lyndsey Wessendorf, Zhanae Seaton, Szymon Zahel, Molly Brown, Alex Hartenstein, and Kurt Manuel for allowing us to share the results of their hard work in LB144. We thank LB student Lindsay Hatter for taking the photographs of the skeletons used in Fig. 3. We heartily thank the LB undergraduate learning assistants and graduate TAs, especially Alicia Bray and Kristi Zurawski, who worked with us to implement and debug the comparative biology stream. We also thank two anonymous reviewers for constructive suggestions and comments.

\section{Appendix 1. Materials Needed, Laboratory Setups, and Detailed Lesson Plans for Each of the Four Weeks of the Comparative Biology Stream}

(NOTE: Included here are the lesson plans that were used to teach this four-week-long lab stream. If you would like 
any of the instructional materials that were used, or if you have any questions about any aspect of the stream and its implementation, please contact one of the authors.)

\section{Overview of the Comparative Biology Lab Stream}

1. Teaching team note: an introduction to phylogeny should occur in class (lecture) prior to this lab stream, and all members of the teaching team should be familiar with the main topics (below) and as a group should discuss the animal phylogenetic trees (including differences between the ones based mainly on molecular vs. morphological characters).

2. Main topics

(a) Comparative morphology

(b) Phylogenetic analysis

(c) Taxonomic classification

3. Major activities

(a) Phylogenetic analysis of five mammals based on skeletal features

(b) Comparative biology (morphology) and phylogenetic analysis of animals representing nine animal phyla

(c) PowerPoint archives and presentations

4. Major products and homework assignments (please note that we use many of the exercises as ungraded (stamped) learning experiences, but this approach could easily be altered)

(a) Research team

- The Little Phylogenetic Analysis: five mammals based on skeletal features

- Thorough PowerPoint of comparative morphology for archival and grading purposes

- The Big Phylogenetic Analysis: nine animal phyla based on morphological features (Note that this exercise can be completed either individually or as a team assignment.)

(b) Individual

- PowerPoint presentation that demonstrates understanding of morphological comparisons across the animal phyla

Week One

a. Materials Needed for Week One

1. Array of organisms for observation in the engagement exercise (see Table 1)

2. Dissecting microscopes as necessary for observations
3. Digital cameras for photomicroscopy (as desired)

4. Computers with MS PowerPoint and photo editing software

5. Fully articulated skeletons of opossum, rabbit, rat, cat and dog and keys

b. Lab Setup for Week One

1. Set up the 18 organisms (see Table 1) at various stations around the lab.

2. Set up the fully articulated skeletons and keys around the lab.

3. Set up dissecting scopes with digital cameras and computers.

c. Lesson Plan-Comparative Biology (CB) Week One

Today's Activities

- Engagement with and exploration of animals in nine phyla (30 min)

- As a team, spend 20 minutes observing and taking notes on the organisms on display. Also, list any observations and/or questions that your team has.

- Goal: Put your observations into a logical framework in the form of a data table. (Students will need to think about and get help on how to do this (what are rows and columns, and why?)). Encourage each group to think about which characters are discriminating for comparison (depends on taxa) and point out that our goal is to discover (make an inference about) shared ancestry.

- Product: data table. Check and stamp each data table in lab.

- Spend 10 minutes debriefing - share contrasting ways to organize the organisms with the class, make sure that they get to a "taxa by characters" table.

- Phylogenetic analysis of five mammals based on skeletal features - interpreting and evaluating phylogenies (60 min)

- Start with a ppt slide of phylogenetic tree from Giese paper (Fig. 1a in (Giese 2005) - overview of how tree is developed, what it shows, and that it is a hypothesis (this example is of an incorrect tree)

- Work through the tree with the students using "If, then, therefore" progression: If the tree is correct, then one of two things must have happened with regard to a particular character (e.g., hair). It could have either evolved independently two times on different branches (not likely), or the evolution of that trait preceded the most recent common ancestor of all taxa with that characteristic. However, by using a tree with a misplaced trait, we can then show that if this is the case, then that trait would have had to have 
been subsequently lost multiple times (on different branches), which is also unlikely. Therefore, the hypothesis itself seems unlikely, at least in regard to this particular trait. Ask questions like "how do we know that the cat is in the wrong place?"

- Point out the locations of the five skeletons and keys (rat, rabbit, cat, dog, and opossum (outgroup)). Provide each student team with a handout showing the 15 possible resolved trees with the opossum as the outgroup (Fig. 4). Assign each team with two trees hypothesizing different phylogenetic relationships for the five taxa.

- Learning Goal: Students will use data (observations) that they record from the skeletons, plus other information that they $\mathrm{know} /$ find out about the biology of the taxa (from www and in table we supply), to argue why they think the data support or do not support each of their two given hypotheses (phylogeny).

- Instructions to students:

- Study the five skeletons and make informal comparisons by noting similarities and differences between them. Each team should then find three to four characters (one per person) that can be used to group taxa together to the exclusion of other taxa. Use the keys to the skeletons to assist your team in finding out what the different bones are and for making your comparisons. Devise a way to score your character (define character states) for each of the five taxa.

- Provide the students with Table 2 that combines Table 3 from Singer et al. (2001) and info that can be found on provided web links to the natural history information for each of the five animals (Michigan Biodiversity Web). Student teams will fill in this table by incorporating the three to four characters that they found by examining the skeletons and others from the www pages.

- Student teams should reflect on which characters of the data set they think might be useful for evaluating phylogenetic hypotheses. Among these could be characters of the animals' physiology (Does it feed the fetus via a placenta?), behavior (Is it a social animal?) and/or ecology (Is it an aquatic animal?).

- Student teams should test their two alternative hypotheses based on whether species share derived characters. You may give more weight to characters that your research team thinks will be more useful for discriminating between the alternative evolutionary hypotheses. Include the physiological, behavioral, and ecological characters in your tests. Use the "If, then, therefore" reasoning demonstrated earlier.

o As a team, write a paragraph that summarizes your work. Defend your preferred hypothesis and justify each node/branch of this phylogenetic tree. Have your paragraph stamped and initialed by your TA.

- Products: data table (Table 2), alternative hypotheses with branches/groupings marked that are supported by evidence and written paragraph.

- Spend 15 minutes debriefing-Have each group present the two trees that they evaluated on an overhead transparency. Each group should indicate which of the two trees they think is better supported by data, and what evidence can be used to support the tree they prefer and why (concentrating on why the other is wrong).

- Making a power point presentation (30 min)

- Do a demo showing the students the basics of how to maneuver in MS PowerPoint (see Appendix 2 for links to examples of student work).

o Have each team start their PowerPoint by making a title slide and inserting the animals' common and scientific names into a blank slideshow.

$\circ$ Every week, each team will add materials to their PowerPoint.

- Product: Before each team leaves lab, have an instructor check that their slideshow has been started and that it has been saved in the appropriate place.

Week Two

A. Materials Needed for Week Two

1. The following animals for observation and/or dissection (Table 1):

(a) Scypha sp. (Sponge; Phylum Porifera)

(b) Hydra sp. (Phylum Cnidaria)

(c) Dugesia tigrina (Brown Planarian; Phylum Platyhelminthes)

(d) Ascaris lumbricoides (Roundworm; Phylum Nematoda); Dissection

(e) Lumbricus terrestris (Earthworm; Phylum Annelida); Dissection

2. Dissecting supplies: pans, pins, and scalpels, gloves, watch glasses, dissection "guidebook," waste bag

3. Dissecting and compound microscopes as necessary for observations

4. Digital cameras for photomicroscopy with appropriate computer software for image capture

5. Computers with MS PowerPoint and photo editing software 
B. Lab Setup for Week Two

1) Set up dissecting and compound scopes with digital cameras and computers.

2) Provide enough dissecting supplies for each team to have two dissections going on at a time.

C. Lesson Plan-Comparative Biology Week Two

Today's Activities

- PowerPoint presentation by instructor introducing main topics (20-30 $\mathrm{min})$

○ Introduction to Comparison of Morphological, Developmental, and Life History Features in Nine Animal Phyla (Table 1) using laboratory observation and guided dissections to compare key organisms representing different phylafive animals this week, five next week.

- We will look at each animal with respect to a set of 11 characteristics (Table 3)-discuss each character a little within a phylogenetic context (e.g., When did the body cavity likely appear on a tree of animals?).

- Lesson on "How To Dissect" for the Roundworm and the Earthworm

- What are the tools?

○ How do we prep the animals?

- How should we cut and pin the animals?

- What should we be looking for?

- Using laboratory observation and guided dissections to compare key organisms representing different phyla (two to two and one-half hours; Table 4)

o For each of the animals that we observe and/or dissect, each research team needs to take a set of four (minimum) to six (preferable) digital photomicrographs that summarizes the features of that organism.

- The data and the observations that each team makes should be used to complete the appropriate parts of the data table (Table 3 ).
- Each of the photomicrographs should be incorporated into your PowerPoint file, and supported by labels, legends, and text that will allow people looking at the pages to understand what it is that they are looking at (or for). Remember that you will need to be able to compare morphology/ systems across phyla, so take/document pictures accordingly.

o Products: Before each team leaves lab, an instructor should check Table 3 and the PowerPoint for progress made.

Week Three

\section{A. Materials Needed for Week Three}

1. The following preserved animals for observation and/or dissection (Table 1):

(a) Anodonta sp. (Freshwater Mussels; Phylum Mollusca)

(b) Cambarus sp. (Crayfish; Phylum Arthropoda); Dissection

(c) Romalea sp. (Grasshopper; Phylum Arthropoda); Dissection

(d) Pisaster sp. (Sea Star; Phylum Echinodermata)

(e) Branchiostoma sp. (Lancelet; Phylum Chordata)

2. Dissecting supplies: pans, pins, and scalpels, gloves, watch glasses, dissection "guidebook," waste bag

3. Dissecting and compound microscopes as necessary for observations

4. Digital cameras for photomicroscopy with appropriate computer software for image capture

5. Computers with MS PowerPoint and photo editing software

B. Lab Setup for Week Three

1. Set up dissecting and compound scopes with digital cameras and computers.

Table 4 Key organisms and actions to be taken

\begin{tabular}{|c|c|c|}
\hline Organism & What we have to look at & What we will do \\
\hline Sponge (Scypha sp., aka Grantia sp.) & $\begin{array}{l}\text { Preserved specimens and prepared slides (near-median } \\
\text { longitudinal section) }\end{array}$ & Observations only \\
\hline Hydra (Hydra sp.) & Live specimens and prepared slides (general structure) & Observations only \\
\hline Brown planarian (Dugesia tigrina) & Live specimens and prepared slides (whole mount and cross section) & Observations only \\
\hline Roundworm (Ascaris lumbricoides) & Preserved specimens and prepared slides (cross sections) & Dissection and observations \\
\hline Earthworm (Lumbricus terrestris) & Preserved specimens and prepared slides (cross sections) & Dissection and observations \\
\hline
\end{tabular}


2. Provide enough dissecting supplies for each team to have two dissections going on at a time

C. Lesson Plan-Comparative Biology Week Three

Today's Activities

- PowerPoint presentation by instructor introducing main topics (20-30 $\mathrm{min})$

- Continuation of Comparison of Morphological, Developmental, and Life History Features in Nine Animal Phyla (Table 1) using laboratory observation and guided dissections to compare key organisms representing different phyla-five animals last week, five this week.

- Reinforce the major features we are looking for in each animal (Table 3) and that they should be thinking within a phylogenetic context.

- Remind the students what they should be trying to accomplish in their work: dissecting the preserved animals, and making observations on preserved animals and prepared slides.

- Look ahead - communicate expectation for:

- PowerPoint file-complete file to be uploaded to course website before the beginning of lab next week, see Online Supplemental Materials for more details of the Assignment.

- PowerPoint presentation - bring your comparative slides to lab next week for your presentations, see Online Supplemental Materials in Appendix 2 for more details of the Assignment.

- Big phylogeny exercise - this exercise can be completed either individually or as a team. Either way, students should be reminded to revisit their notes from class and lab in terms of how to evaluate alternative trees. It is especially important to remind the students to revisit the skeleton exercise from week one of the $\mathrm{CB}$ stream and for the instructor to go over the most common mistakes/misconceptions student teams had from this exercise. Remind them to map characters, to include a clear key, and to discuss which tree is better supported and WHY.
- Lesson on "How To Dissect" for the Grasshopper and the Crayfish

$\circ$ What are the tools?

$\circ$ How do we prep the animals?

$\circ$ How should we cut and pin the animals?

○ What should we be looking for?

- Using laboratory observation and guided dissections to compare key organisms representing different phyla (two to two and one-half hours; Table 5)

o For each of the animals that we observe and/ or dissect, each research team needs to take a set of four (minimum) to six (preferable) digital photomicrographs that summarizes the features of that organism.

- The data and the observations that each team makes should be used to complete the appropriate parts of the data table (Table 3).

- Each of the photomicrographs should be incorporated into your PowerPoint file, and supported by labels, legends, and text that will allow people looking at the pages to understand what it is that they are looking at (or for). Remember that you will need to be able to compare morphology/systems across phyla, so take/ document pictures accordingly.

- Products: Before each team leaves lab, an instructor should check Table 3 and the PowerPoint for completion (Table) progress made (PowerPoint).

\section{Week Four}

A. Materials Needed for Week Four

1. Computer with MS PowerPoint, overhead projector, and screen

B. Lab Set-up for Week Four

1. Set up lab with screen, projector, and computer for student presentations

Table 5 Key organisms and actions to be taken

\begin{tabular}{lll}
\hline Organism & What we have to look at & What we will do \\
\hline Freshwater mussel (Anodonta sp.) & Preserved specimens and prepared slide (gill cross-section) & Observations only \\
Grasshopper (Romalea sp.) & Preserved specimens & Dissection and observations \\
Crayfish (Cambarus sp.) & Preserved specimens & Dissection and observations \\
Sea Star (Pisaster sp.) & Dissected preserved specimens and prepared slides & Observations only \\
Lancelet (Branchiostoma sp.) & Preserved specimens and prepared slides & Observations only \\
\hline
\end{tabular}




\section{Lesson Plan-Comparative Biology Week Four}

Today's Activities

- No PowerPoint presentation by instructor; load student presentations on computer.

- Each group should present together, but each student presents their two comparison slides individually, talking for three to five minutes about the animals in a phylogenetic context.

\section{Appendix 2. Brief Descriptions of and Links to Student Work Available as Online Supplemental Materials}

1. Electronic notebook (PowerPoint file) made by a student team in LB144 F06. In fall semester 2006, the student notebook took the form of a PowerPoint file that contained all of the student pictures taken during weeks two and three. Each team of three students was required to establish a collection of four to six slides per animal (some with multiple photos per slide), with annotation. The linked file represents one of the best of the student notebooks: http://www. msu.edu/user/jimsmith/evoo1.ppt

2. Electronic notebook (PowerPoint file) made by students in LB144 F08. In fall semester 2008, each individual student was required to organize a set of two slides that would form the basis of an oral presentation that they would give to the class during week four. Each of these PowerPoint slides was to include multiple photos that demonstrated student understanding of similarities and differences in at least three of the animal phyla that were studied: http://www. msu.edu/user/jimsmith/evoo2.ppt

\section{References}

Avise JC. Molecular markers, natural history, and evolution. Sunderland: Sinauer Associates; 2004.

Baum DA, Smith SD, Donovan SSS. Evolution: the tree-thinking challenge. Science. 2005;310:979-80.

Bilardello N, Valdés N. Constructing phylogenies. Am Biol Teach. 1998;60:369-73.

Campbell NA, Reece JB. Biology. 6th ed. Menlo Park: Benjamin/ Cummings; 2002.

Colburn A. An inquiry primer. Sci Scope. 2000;23:42-4.

Dobzhansky T. Nothing in biology makes sense except in the light of evolution. Am Biol Teach. 1973;35:125-29.

Elson LM. The zoology coloring book. New York: Harper-Collins; 1982.

Field KG, Olsen GJ, Lane DJ, Giovannoni SJ, Ghiselin MT, Raff EC, et al. Molecular phylogeny of the animal kingdom. Science. 1988;239:748-53.

Freeman S. Biological science. 2nd ed. Upper Saddle River: Pearson/ Prentice Hall; 2005.

Futuyma DJ. Wherefore and whither the naturalist? Am Nat. 1998;151:1-6.

Giese AR. Using inquiry and phylogeny to teach comparative morphology. Am Biol Teach. 2005;67:412-7.

Grant PR. What does it mean to be a naturalist at the end of the twentieth century? Am Nat. 2000;155:1-12.

Johnson DW, Johnson R, Smith K. Advanced cooperative learning. 3rd ed. Edina: Interactive Book; 1998.

McGlynn TP. Natural history education for students heading into the century of biology. Am Biol Teach. 2008;70:109-11.

Morgan JG, Carter MEB. Investigating biology. 5th ed. New York: Pearson/Benjamin Cummings; 2005.

Novick LR, Catley KM. Understanding phylogenies in biology: the influence of a Gestalt perceptual principle. J Exp Psychol Appl. 2007; 13:197-223.

Rust TG. A guide to biology lab. 3rd ed. San Antonio: Southwest Educational; 1983.

Singer F, Hagen JB, Sheehy RR. The comparative method, hypothesis testing \& phylogenetic analysis - an introductory laboratory. Am Biol Teach. 2001;63:518-23.

Wilson EO. On the future of conservation biology. Conserv Biol. 2000;14:1-3.

Wilterding JH, Luckie DB. Increasing student-initiated active learning with investigative "streams:" a molecular biology example. J Coll Sci Teach. 2002;31:303-7. 